\title{
Discriminant Function Using Fisher's Approach For Three Multivariate Normal Populations
}

\author{
${ }^{1}$ Khawla Mustafa Sadek and ${ }^{2}$ BasimaIdris Ismail \\ 1,2Department of Mathematical Education College Mosul University, Iraq
}

Correspondence Author: Khawla Mustafa Sadek, Department of Mathematical Education College Mosul University, Iraq

Received date: 23 February 2018, Accepted date: 1 April 2018, Online date: 15 April 2018

Copyright: (C) 2018 Khawla Mustafa Sadek and BasimaIdris Ismail. This is an open-access article distributed under the terms of the Creative Commons Attribution License, which permits unrestricted use, distribution, and reproduction in any medium, provided the original author and source are credited.

\begin{abstract}
Background: Discriminant analysis is used in situations where the populations or groups are known apriori. one of the aims of discriminant analysis is to classify an observation, or several observations, in to these known populations, In this case we know that it or they have come from exatly one of those populations but we do not know from which. The other aim is to interpret the differences between the populations, in terms of a few linear functions of the orginal variables. In the following we use classification instead of discrimination although kendall (1966) has extensive notes the terminology.
\end{abstract}

Key words: Multivarate Normal distributiong, Baye'sTheorm, DiscriminatFunction, Fisher's Approach.

Abstract:

We consider a classification of $q$ groupe. Let $y \in\{1,2, \ldots, q\}$ be adependent variable associated with each object or observation.

Suppose that the group conditional population densities $\mathrm{f}_{\mathrm{i}}(\mathrm{x})=\mathrm{f}(\mathrm{x} / \mathrm{y}=\mathrm{i})$ and the population prior $\rho_{\mathrm{i}}$ are unknown. The Baye'stheorm may be used to express the posterior probability of population $\mathrm{i}$ given observation vector $\mathrm{x}$.

$$
f(i / x)=\frac{\rho_{i} f_{i}(x)}{\sum_{\ell=1}^{q} \rho_{\ell} f_{\ell}(x)}
$$

If the distribution of $\mathrm{x}$ is continuous, it is sufficient to replace $\mathrm{x}_{\mathrm{o}}$ by a $\delta$ neighborhood No $\left(\mathrm{x}_{\mathrm{o}}\right)$ of $\mathrm{x}_{\mathrm{o}}$ and to tend $\delta \rightarrow 0$. Baye's rule predicts the population of an observation vector $\mathrm{x}$ by that with the highest posterior probability $\max \rho_{\mathrm{i}} \mathrm{f}_{\mathrm{i}}(\mathrm{x})=\rho_{\mathrm{i}} \mathrm{f}_{\mathrm{i}}(\mathrm{x}) \Rightarrow x \in \mathrm{G}_{\mathrm{i}}$.

Fisher's Approach when the $\pi_{\mathrm{i}}$ distributed multivariate normal have the same covariance matrix $\sum$, the (sample) linear discriminant Function's $\grave{b}_{\mathrm{i}} \mathrm{X}$, $\mathrm{i}=1, \ldots$, $\mathrm{m}=\min \{\mathrm{p}, \mathrm{q}-1\}$ were introduced by fisher (1936) achieve a good separation such functions were found by condsidering the linear combination bx that maximizes the ratio of the between- group sum of squares to the within- group sum of squares.

Objective:

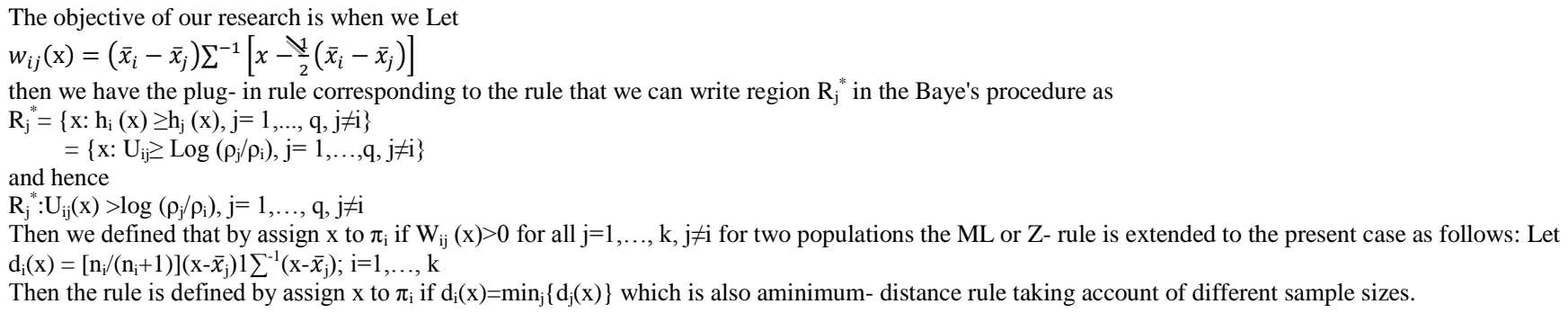

Fisher's Approach:

Suppose that (q) population $\pi_{\mathrm{i}}$ have the same covariance matrix $\sum$. suppose that we have the $\mathrm{n}_{\mathrm{i}}$ samples from $\pi_{\mathrm{i}}$ populations. Let us denote the between- group and within- group $\mathrm{SS}_{\mathrm{b}}$ matrices by:

$\mathrm{S}_{\mathrm{b}}=\mathrm{n}_{1}\left(\bar{x}^{(1)}-\bar{x}\right)\left(\bar{x}^{(1)}-\bar{x}\right)+\ldots+\mathrm{n}_{\mathrm{q}}\left(\bar{x}^{(q)}-\bar{x}\right)\left(\bar{x}^{(q)}-\bar{x}\right)$,

$\mathrm{S}_{\mathrm{w}}=\left(\mathrm{n}_{1}-1\right) \mathrm{S}^{(1)}+\ldots+\left(\mathrm{n}_{\mathrm{q}}-1\right) \mathrm{S}^{(\mathrm{q})}$

Now consider a linear combination of $\mathrm{X}_{\mathrm{i}}$ :

$\mathrm{Z}=\mathrm{b}_{1} \mathrm{x}_{1}+\ldots+\mathrm{b}_{\mathrm{p}} \mathrm{x}_{\mathrm{p}}=\grave{b} \mathrm{x}$

where $b=\left(b_{1}, \ldots, b_{p}\right)$. The between- group and within- group sums of squares of $Z$ are expressed as $\grave{b} S_{b} b$ and $S_{w} b$. As a coefficient vector $b$ with a good separation of q groups, Fisher proposed to maximizing the ratio

Optimum solutions are obtained by considering the characteristic equation

$$
\ell=\frac{\grave{b} S_{b} b}{\grave{b} S_{w} b}
$$

$\mathrm{S}_{\mathrm{b}} \mathrm{b}_{\mathrm{i}}=\ell_{i} S_{w} b_{i}, \grave{b}_{i} S_{w} b_{i}=n S_{i j}$

where $\ell_{1}>\cdots>\ell_{m} \geq 0$ are possibly non zero characteristic roots of $\mathrm{S}_{\mathrm{b}} S_{w}^{-1} \mathrm{~m}=\min \{\mathrm{p}, \mathrm{q}-1\}, \delta_{\mathrm{ij}}$ is kronecker'sdalta, and $\delta_{\mathrm{ii}}=1, \delta_{\mathrm{ij}}=0$ for $\mathrm{i} \neq \mathrm{j}$.

Now we have $\mathrm{m}$ (meaningful) functions $\mathrm{Z}_{\mathrm{i}}=\grave{\mathrm{b}}_{\mathrm{i}} \mathrm{x}, \mathrm{i}=1, \ldots, \mathrm{m}$ called canonical discriminant functions. 
Know if we extended the decision- theoretic approach for two populations to that for (q) populations, Let $\pi_{1}, \ldots, \pi_{\mathrm{q}}$ be q populations with density functions $f_{1}(x), \ldots, f_{q}(x)$ respectively. we wish to divide the sample space $R^{p}$ in to $q$ mutually exclusive and exhaustive regions $R_{1}, \ldots, R_{q}$.

If an observation falls in to $R_{i}$, we say that it comes from $\pi_{\mathrm{i}}$. Let $\mathrm{c}(\mathrm{i} / \mathrm{j})$ be aloss when a $\pi_{\mathrm{j}}$ observation is assigned to $\mathrm{R}_{\mathrm{i}}$, and $\mathrm{c}(\mathrm{i} / \mathrm{i})=0$.

Then the risk is

$$
\sum_{\mathrm{i}=1}^{\mathrm{q}} \sum_{\mathrm{j} \neq \mathrm{i}} \mathrm{c}(\mathrm{j} / \mathrm{i}) \rho_{\mathrm{i}} \mathrm{p}(\mathrm{j} / \mathrm{i})
$$

and for an equalvar- covariance $\sum_{1}=\sum_{2}=\sum_{3}=\ldots=\sum$ we take three cases $\sum_{1}=\sum_{2}=\sum_{3}=\sum$

$$
\mathrm{U}(\mathrm{x})=\log \left(\frac{\mathrm{f}\left(\mathrm{x}_{1}, \theta\right)}{\mathrm{f}\left(\mathrm{x}_{2}, \theta\right)}\right)
$$

$=\mathrm{B}_{\mathrm{o}}+\grave{\mathrm{B}}_{1} \mathrm{x}$

$$
=\left(\mu_{1}-\mu_{2}\right) \sum^{-1}\left[x-\frac{1}{2}\left(\mu_{1}-\mu_{2}\right)\right]
$$

where

$\mathrm{B}_{0}=\frac{-1}{2}\left(\mu_{1}-\mu_{2}\right) \sum^{-1}\left(\mu_{1} \neq \mu_{2}\right)$

$\mathrm{B}_{1}=\sum^{-1}\left(\mu_{1}-\mu_{2}\right)$

Know the same rule for three variables $\mathrm{x}_{1}, \mathrm{x}_{2}, \mathrm{x}_{3}$

$\mathrm{u}(\mathrm{x})=\log \left[\left(\frac{\mathrm{f}\left(\mathrm{x}_{1}, \theta\right)}{\mathrm{f}\left(\mathrm{x}_{2}, \theta\right)}\right) / \mathrm{f}\left(\mathrm{x}_{3}, \theta\right)\right]$

$\mathrm{u}(\mathrm{x})=\log \mathrm{f}\left(\mathrm{x}_{1}, \theta\right)-\log \mathrm{f}\left(\mathrm{x}_{2}, \theta\right)-\log \mathrm{f}\left(\mathrm{x}_{3}, \theta\right)$

$\mathrm{u}(\mathrm{x})=\left(\mu_{1}-\mu_{2}\right) \sum^{-1}\left[-\frac{1}{2}\left(\mu_{1}+\mu_{2}\right)\right]-\log \mathrm{f}\left(\mathrm{x}_{3}, \theta\right)$

$=\left(\mu_{1}-\mu_{2}\right) \mathbb{\Sigma}^{-1}\left[x-\frac{1}{2}\left(\mu_{1}+\mu_{2}\right)\right]+\frac{1}{2} \grave{x} \Sigma^{-1} x-\grave{x} \Sigma^{-1} \mu_{3}+\frac{1}{2} \grave{\mu}_{3} \Sigma^{-1} \mu_{3}$

$\left.=\left(\mu_{1}-\mu_{2}-\mu_{3}\right) \sum \backslash x-\frac{1}{2}\left(\mu_{1}+\mu_{2}+\mu_{3}\right)\right]+\frac{1}{2} \grave{x} \sum^{-1} x$

$\therefore$ The discriminant functions of the three variables

$=\grave{x} \sum^{-1}\left(\mu_{1}-\mu_{2}-\mu_{3}\right)+\frac{1}{2} \grave{x} \Sigma^{-1} x$

know we saw that in the two populations if:

$\frac{\mathrm{f}\left(\mathrm{x}_{1}, \theta\right)}{\mathrm{f}\left(\mathrm{x}_{2}, \theta\right)} \geq k \Rightarrow x \mathrm{falls}$ in the first population

$\frac{\mathrm{f}\left(\mathrm{x}_{1}, \theta\right)}{\mathrm{f}\left(\mathrm{x}_{2}, \theta\right)}<k \Rightarrow x \mathrm{falls}$ in the second population

But here we have two $k_{9} k_{1} \& k_{2}$

The first onedelate to divide $\mathrm{f}\left(\mathrm{x}_{1}, \theta\right) / \mathrm{f}\left(\mathrm{x}_{2}, \theta\right)$ and the second delate to divide $\mathrm{f}\left(\mathrm{x}_{1}, \theta\right) / \mathrm{f}\left(\mathrm{x}_{3}, \theta\right)$

$\frac{\mathrm{f}\left(\mathrm{x}_{1}, \theta\right)}{\mathrm{f}\left(\mathrm{x}_{2}, \theta\right)} \geq k_{1} \Rightarrow x \mathrm{falls}$ in $\pi_{1}$

$\frac{\mathrm{f}\left(\mathrm{x}_{1}, \theta\right)}{\mathrm{f}\left(\mathrm{x}_{2}, 0\right)}<k_{1} \Rightarrow x$ falls in $\pi_{2}$

$\mathrm{f}\left(\mathrm{x}_{2}, \theta\right)$

$\frac{\mathrm{f}\left(\mathrm{x}_{1}, \theta\right)}{\mathrm{f}\left(\mathrm{x}_{3}, \theta\right)} \geq k_{2} \Rightarrow x$ falls in $\pi_{1}$

$\frac{\mathrm{f}\left(\mathrm{x}_{1}, \theta\right)}{\mathrm{f}\left(\mathrm{x}_{3}, \theta\right)}<k_{2} \Rightarrow x$ falls in $\pi_{3}$

we have the following:

$\pi_{1}$

$$
\mathbf{k}_{\mathbf{1}}
$$

$\boldsymbol{\pi}_{2}$

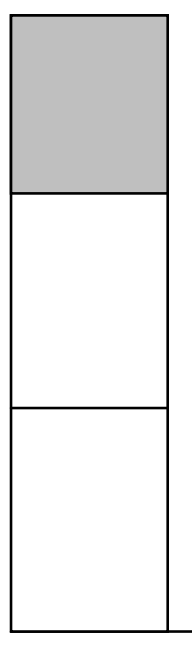

$\pi_{3}$

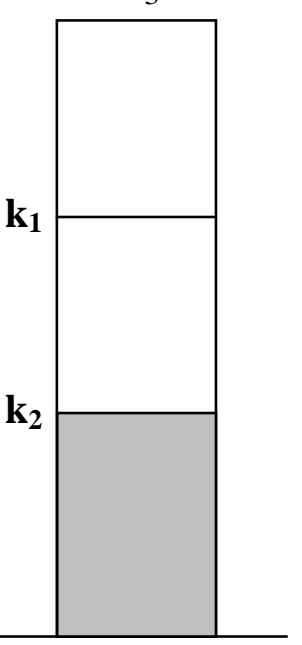

$\mathrm{A}: \frac{\mathrm{f}\left(\mathrm{x}_{1}, \theta\right)}{\mathrm{f}\left(\mathrm{x}_{2}, \theta\right)} \geq k_{1}$

$(\mathrm{A} \cap \mathrm{C})=x$ falls in $\pi_{3}$ if $x \geq \mathrm{k}_{1}$

B: $\frac{\mathrm{f}\left(\mathrm{x}_{1}, \theta\right)}{\mathrm{f}\left(\mathrm{x}_{2}, \theta\right)}<k_{1}$ Then $x$ falls in $\pi_{2}$

$\mathrm{B}-\mathrm{C}=x$ falls in $\pi_{2}$ if $\mathrm{k}_{2}<x<\mathrm{k}_{1}$

C: $\frac{\mathrm{f}\left(\mathrm{x}_{1}, \theta\right)}{\mathrm{f}\left(\mathrm{x}_{2}, \theta\right)} \geq k_{2}$

D: $\frac{\mathrm{f}\left(\mathrm{x}_{1}, \theta\right)}{\mathrm{f}\left(\mathrm{x}_{2}, \theta\right)}<k_{2}$ Then $x$ falls in $\pi_{3}$

Now return to covariance matrix we suppose that:

$S=\left[\begin{array}{lll}S_{11} & S_{12} & S_{13} \\ S_{21} & S_{22} & S_{23} \\ S_{31} & S_{32} & S_{33}\end{array}\right]$

For two groups and for three variables. Then we write the covariance matrix between S and A which is the variance of the third group and denote to it by $\mathrm{L}$, since the variance- covariance between the group $\mathrm{A}$ and $\mathrm{B}$ and group $\mathrm{C}$ is: 
$\mathrm{L}=\left[\begin{array}{lll}\ell_{11} & \ell_{12} & \ell_{13} \\ \ell_{21} & \ell_{22} & \ell_{23} \\ \ell_{31} & \ell_{32} & \ell_{33}\end{array}\right]$

we use it to calculate "fisher discriminant function"

$\mathrm{Z}=\grave{\mathrm{x}} \mathrm{L}^{-1}\left(\overline{\mathrm{x}}_{1}^{(\mathrm{i})}-\overline{\mathrm{x}}_{2}^{(\mathrm{i})}-\overline{\mathrm{x}}_{3}^{(\mathrm{i})}\right)+\frac{1}{2} \grave{\mathrm{x}} \mathrm{L}^{-1} \mathrm{x}$

$=\left(\begin{array}{lll}\mathrm{x}_{1} \mathrm{x}_{2} \mathrm{x}_{3}\end{array}\right)\left[\begin{array}{lll}\ell_{11} & \ell_{12} & \ell_{13} \\ \ell_{21} & \ell_{22} & \ell_{23} \\ \ell_{31} & \ell_{32} & \ell_{33}\end{array}\right]^{-1}\left[\begin{array}{ccc}\overline{\mathrm{x}}_{1}^{(\mathrm{i})} & -\overline{\mathrm{x}}_{2}^{(\mathrm{i})} & -\overline{\mathrm{x}}_{3}^{(\mathrm{i})} \\ \overline{\mathrm{x}}_{1}^{(\mathrm{i})} & -\overline{\mathrm{x}}_{2}^{(\mathrm{i})} & -\overline{\mathrm{x}}_{3}^{(\mathrm{i})} \\ \overline{\mathrm{x}}_{1}^{\mathrm{i})} & -\overline{\mathrm{x}}_{2}^{\mathrm{i})} & -\overline{\mathrm{x}}_{3}^{\mathrm{i})}\end{array}\right]$

$$
+\frac{1}{2}\left(\mathrm{x}_{1} \mathrm{x}_{2} \mathrm{x}_{3}\right)\left[\begin{array}{lll}
\ell_{11} & \ell_{12} & \ell_{13} \\
\ell_{21} & \ell_{22} & \ell_{23} \\
\ell_{31} & \ell_{32} & \ell_{33}
\end{array}\right]^{-1}\left(\begin{array}{l}
\mathrm{x}_{1} \\
\mathrm{x}_{2} \\
\mathrm{x}_{3}
\end{array}\right)
$$

Conclusion:

Fisher's Approach us Baye's method which is reduced to the maximum likelihood classification rule which is use to discriminant about two population can be extend to acted with three or more populations with certain sample space.

This approach is very usefull when we use the quntitivevaribles having acertain chooses such that good, very good, exlent or... ete.

Then we can say that fisher's approach is very usefull in our life to act with many different ways.

\section{REFERENCES}

Anderson, T.W., 2003. "Introduction to Multivariate Statistical Analysis" $3^{\text {rd }}$ ed, Wiley- Hoboken, N. J. (41, 161, 320, 326, 6, 334, 511).

Arnold, S.F., 1981. "The Theory of Linear Models and Multivariate Analysis", Wiley- Hoboken, N. J. (154).

Kendall, M.G., 1966. "Discrimination and Classification, In Multivariate Analysis" (P. R. Krichnaiah, ed.). Academic press, New York (249).

Rao, C.R., 1973. "Linear Statistical Inference and It's Applications", $2^{\text {nd }}$ ed, Wiley, New York, $(312,503,505)$.

Srivatava, M.S., 2007. "Multivariate Theory Analyzing High Dimensional Data", J. Japan statist. Soc: 37, 53-86. (279, 280).

Takeuchi, K. and A. Takemura, 1988. "Some Results on Univariate and Multivariate Cornish- Fisher Expansion: Algehraic Properties and Validity" Sankhyā A, 50, 111-136. (130).

Yasunori Fujikoshi and Vladimir V. Ulyanov and Ryoichi Shimizu, 2011. " Multivariate Statistics High- Dimensional and Large- Sample Approximations". 